\title{
Vertebral Osteomyelitis Due to Granulicatella Adiacens, a Nutritionally Variant Streptococci
}

\author{
Jonathan York $^{1}$, Christian Fisahn ${ }^{2}$, Jens Chapman ${ }^{3}$ \\ 1. Neurosurgery, Swedish Neuroscience Institute 2. Orthopedic Surgery, Swedish Neuroscience Institute \\ 3. Orthopedics Spine Surgery, Swedish Neuroscience Institute
}

$\square$ Corresponding author: Christian Fisahn, christian.fisahn@swedish.org

Disclosures can be found in Additional Information at the end of the article

\section{Abstract}

Vertebral osteomyelitis is a common pathology affecting the spine. We present the case of a 46year-old male who was diagnosed with progressive L2 vertebral osteomyelitis due to a rare pathogen, Granulicatella adiacens. Granulicatella adiacens is part of the normal body flora and is often difficult to culture on traditional mediums. The patient required a lateral corpectomy and posterior fixation for spinal stabilization and source control.

Categories: Neurosurgery

Keywords: spine, osteomyelitis, granulicatella adiacens, streptococci, bacterial infection

\section{Introduction}

Vertebral osteomyelitis (VO) is a common entity encountered in a neurosurgical practice. VO is characterized by an acute or recurrent infection of the spine and subsequent inflammatory destruction of the bone. The most common pathogen is Staphylococcus aureus, followed by Escherichia coli [1]. Progressive destruction of the anterior spinal elements can lead to instability, necessitating surgical intervention for anterior column support and spinal stabilization as well as obtaining source control. Herein, we present the rare case of a 46-yearold male who was diagnosed with an unusual organism, Granulicatella adiacens, a subtype of nutritionally variant streptococci (NVS).

\section{Case Presentation}

A 46-year-old male presented with progressively worsening low back pain. His pain began after a fall onto his buttocks three weeks prior to his initial evaluation. His symptoms were

Received 09/06/2016 Review began 09/09/2016 Review ended 09/28/2016 Published 09/28/2016

\section{C) Copyright 2016}

York et al. This is an open access article distributed under the terms of the Creative Commons Attribution License CC-BY 3.0., which permits unrestricted use, distribution, and reproduction in any medium, provided the original author and source are credited. aggravated by movement and alleviated by lying flat. He had little alleviation of his symptoms with over-the-counter anti-inflammatories. He denied any lower extremity pain, numbness, or weakness. His medical history was notable for diabetes and smoking. He denied illicit drug use. He did endorse undergoing a dental procedure approximately one month prior to evaluation. A written informed consent is not necessary for single case reports at our institution (Swedish Institutional Review Board).

On physical examination, he was exquisitely tender to palpation in his upper lumbar spine. He frequently shifted positions to find comfort, and he preferred to lie down. He had no lower extremity (LE) weakness, numbness, or saddle anesthesia. 


\section{Cureus}

Initial lumbar spine computed tomography (CT) obtained in the Emergency Department revealed a subtle irregularity of the L2 inferior endplate. Magnetic resonance imaging (MRI) of the lumbar spine again showed erosion of the L2 inferior endplate as well as diffuse L2 bone marrow edema, suggestive of either recent trauma or infection. MRI with contrast revealed small foci of enhancement of the L2 inferior endplate, as seen in Figures 1-3.

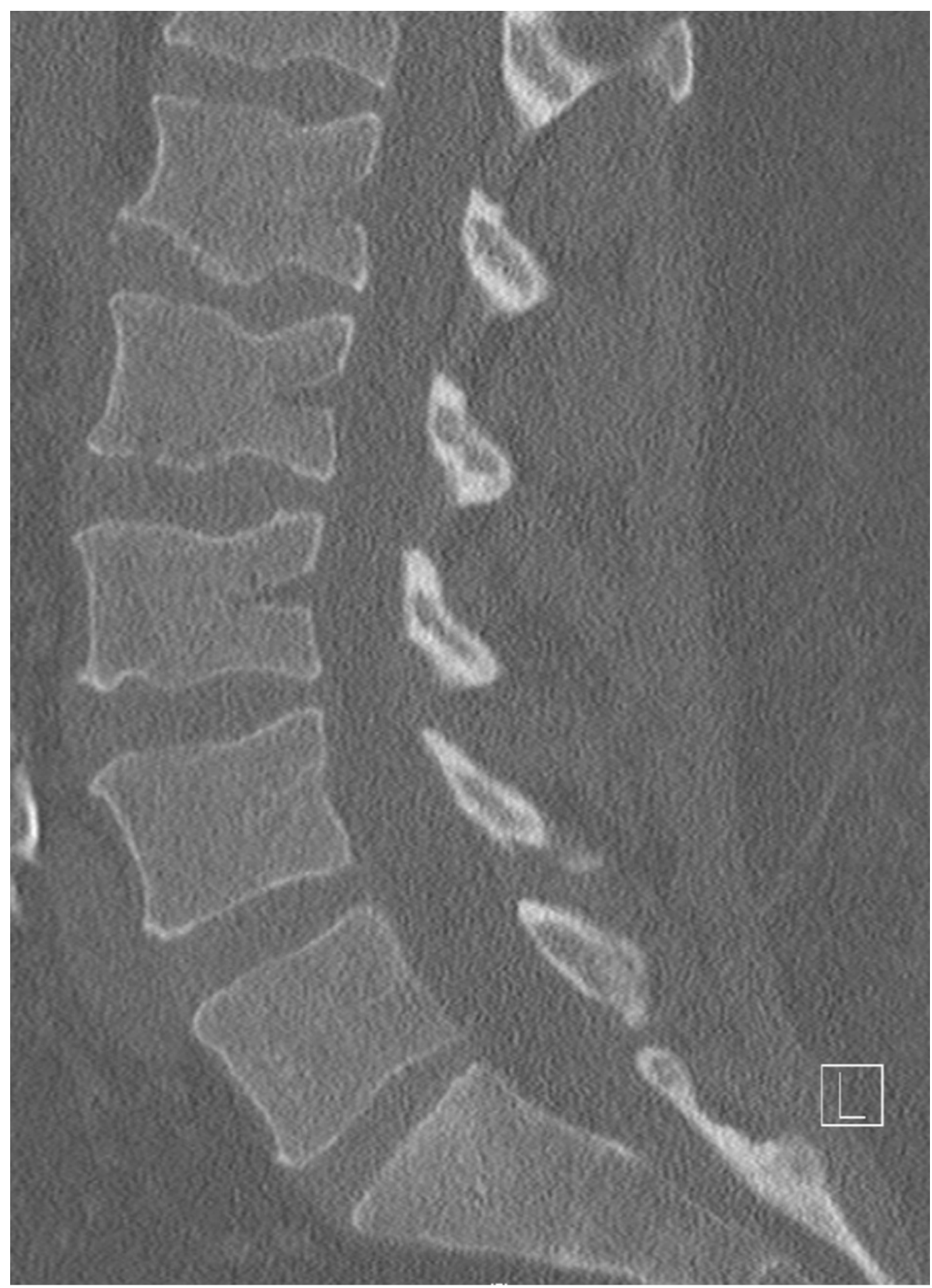

FIGURE 1: Initial CT revealing subtle irregularity of inferior endplate of L2. 


\section{Cureus}

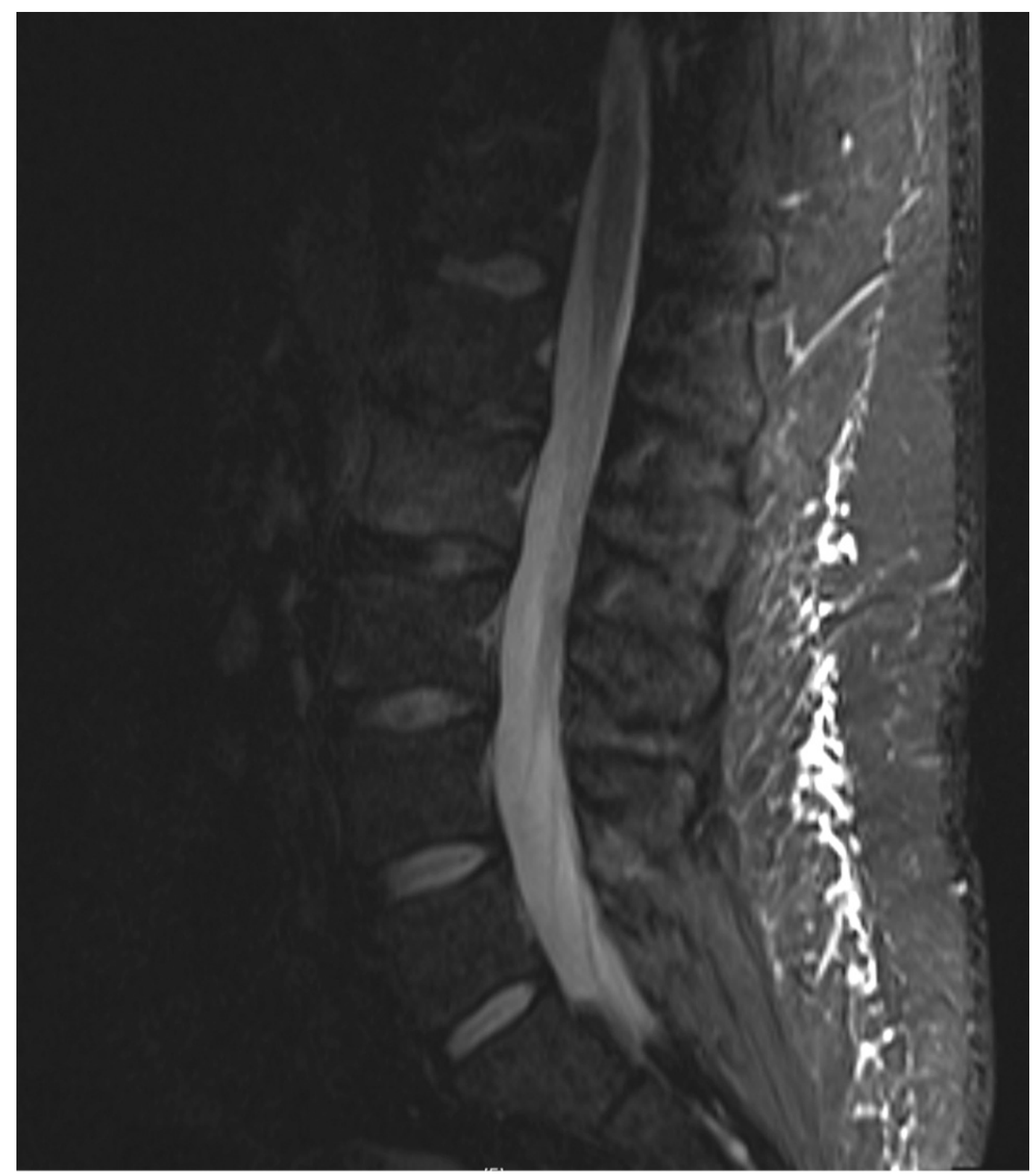

FIGURE 2: Initial MRI with contrast showing increased STIR signal change indicating diffuse bone marrow edema.

STIR - short T1 inversion recovery 


\section{Cureus}

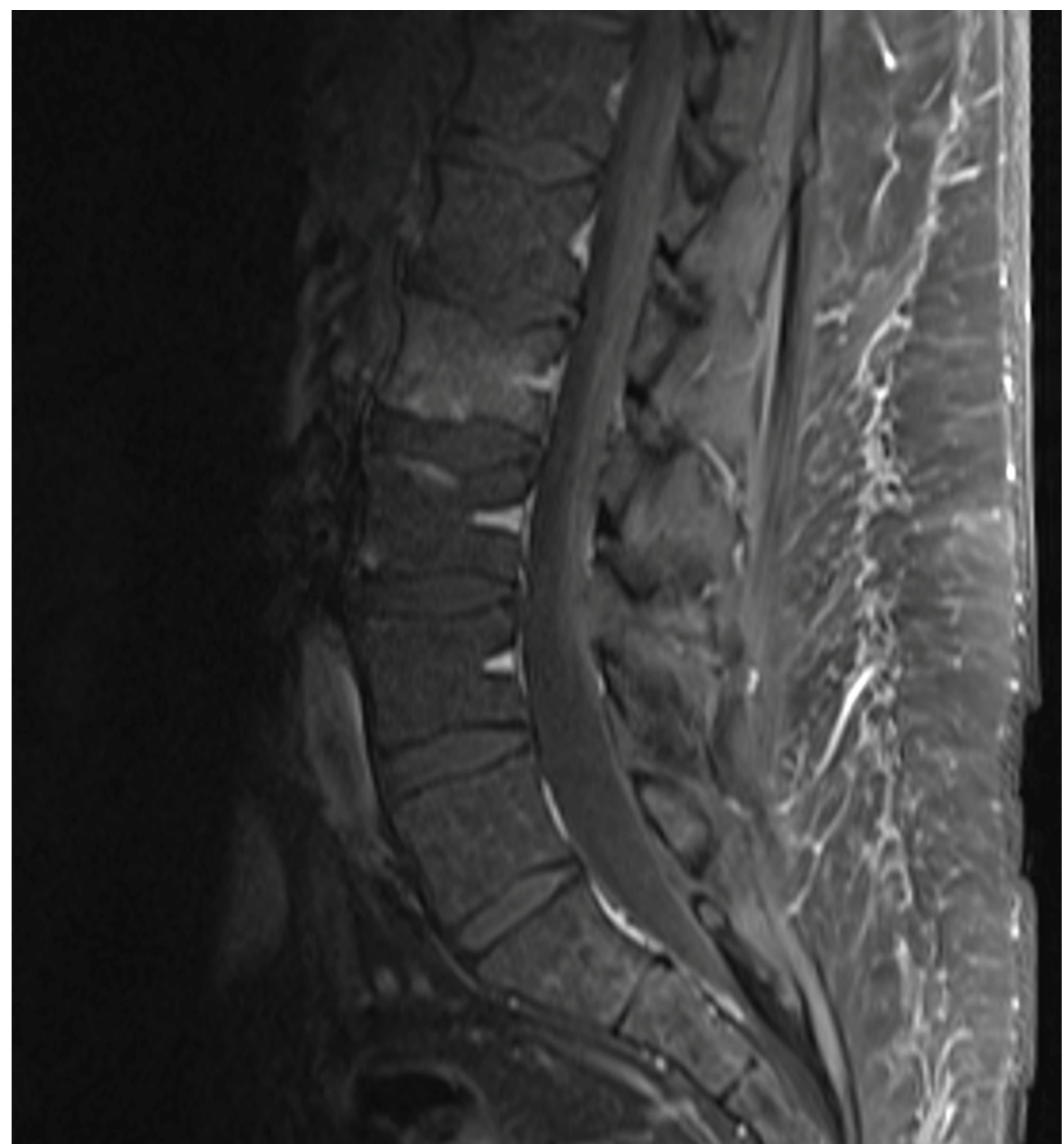

\section{FIGURE 3: Initial MRI without contrast demonstrating foci of enhancement within the inferior L2 vertebral body.}

His laboratory studies revealed a white blood cell (WBC) of 18,000, erythrocyte sedimentation rate (ESR) 18, and a C-reactive protein (CRP) of 3.39. Peripheral blood cultures were negative. The patient was discharged with a thoracolumbosacral orthosis (TLSO) brace and instructions to return for repeat lab work and follow-up in clinic.

The patient returned to the clinic one month later. He complained of continued, severe low back pain that significantly diminished his ability to ambulate. He also endorsed new bilateral lower extremity radiculopathy. The patient had attempted conservative measures with bed rest, the TLSO brace, and oral narcotics but had achieved only minimal pain relief.

Repeat laboratory studies revealed an increased leukocytosis with WBC 20,600, an increased ESR to 29, and decreased CRP to 1.17. Repeat MRI and CT revealed a progression of the L2 central lytic lesion, as seen in Figures 4-5. 


\section{Cureus}

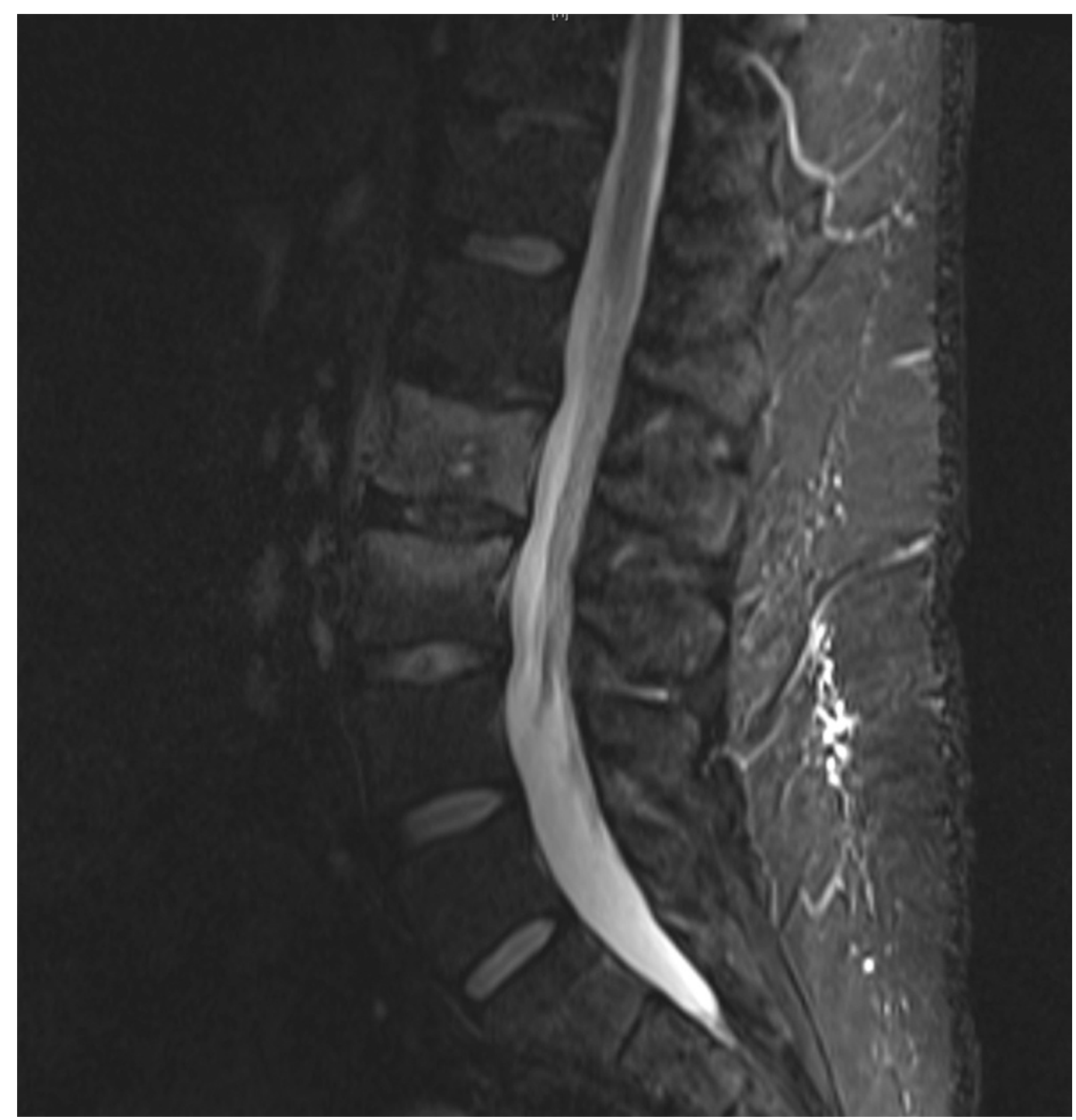

FIGURE 4: Follow-up MRI demonstrating increased signal change within the L2 vertebral body. 


\section{Cureus}

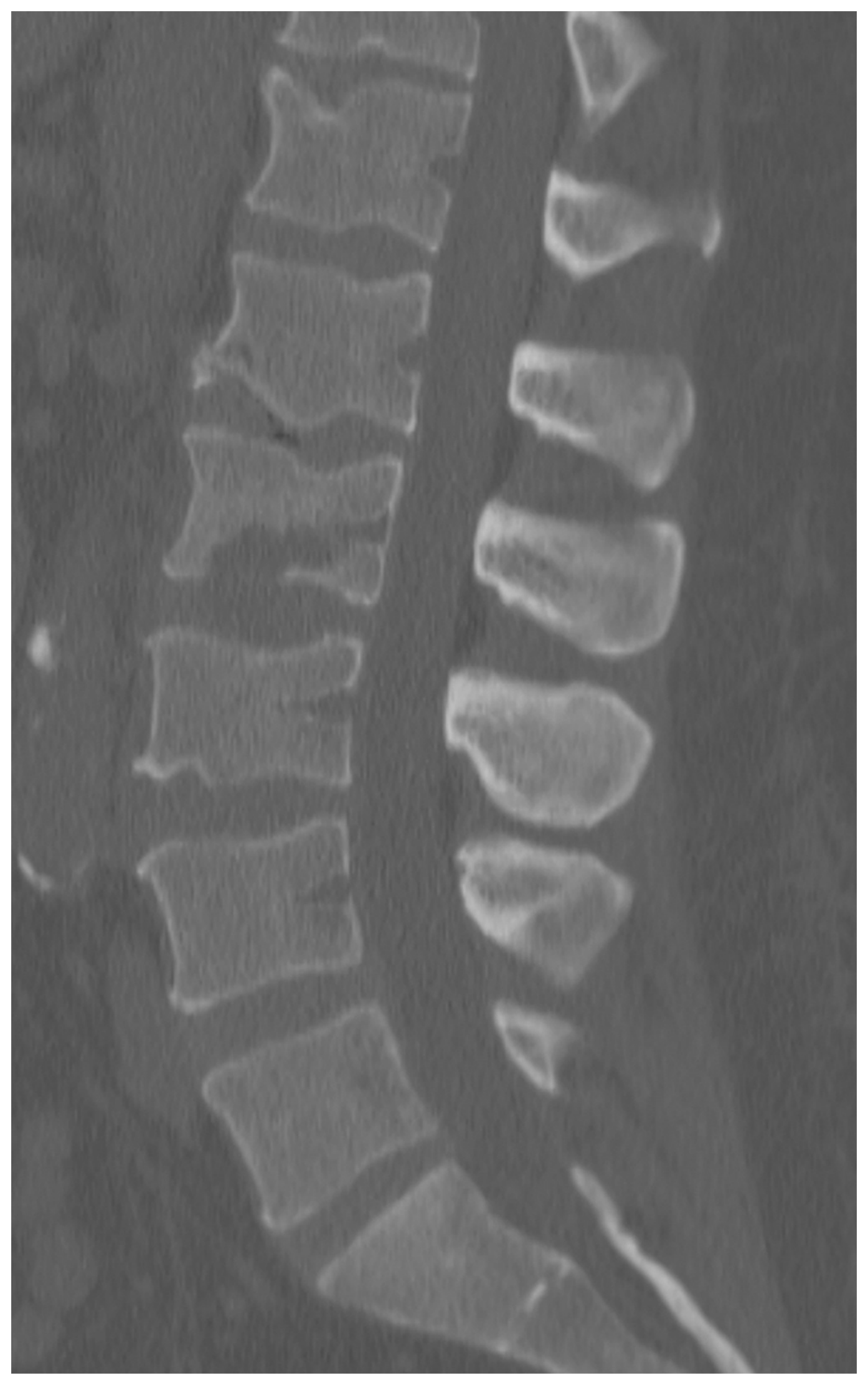

FIGURE 5: Follow-up CT demonstrating a significant progression of osseous destruction of the L2 vertebral body. 


\section{Cureus}

The patient was admitted to hospital for definitive management. He underwent a CT-guided biopsy of the L2 vertebral body, which revealed Granulicatella adiacens on polymerase chain reaction (PCR). He subsequently underwent posterior decompression and fixation from L1 to L3 followed by L2 direct lateral corpectomy with the placement of an interbody cage. His postoperative film revealed appropriate resection of lytic L2 segments with complete neural element decompression (Figure 6). His intraoperative specimens did not reveal a positive pathogen on PCR but did produce NVS on culture, consistent with Granulicatella adiacens.

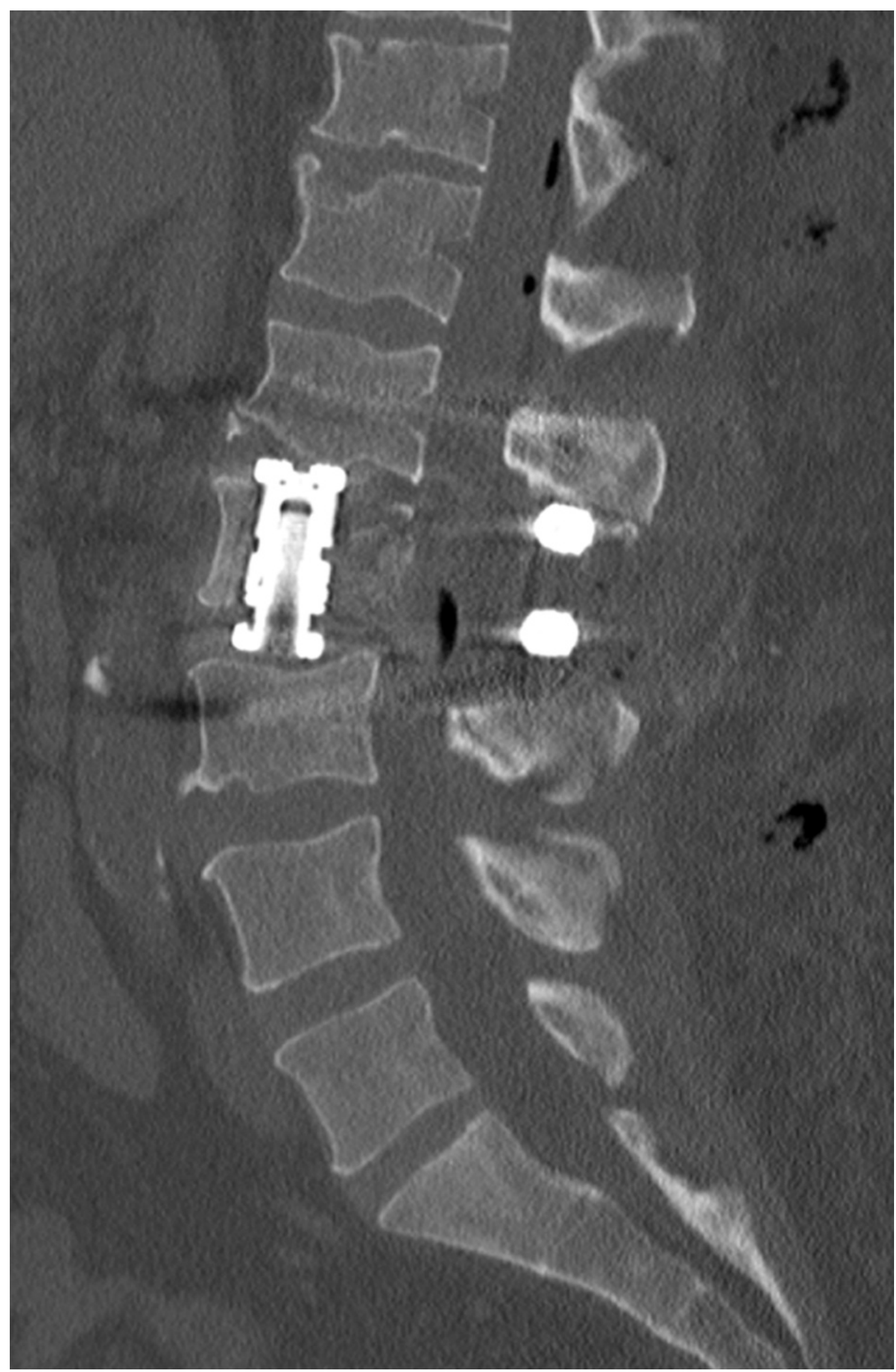


Preoperatively, the patient was started on vancomycin and ceftazidime after his biopsy. His antibiotics were narrowed to vancomycin after his surgical procedure. He was discharged to a skilled nursing facility on postop day six with plans for six weeks of IV antibiotics per the infectious disease team.

The authors report no conflict of interest concerning the materials or methods used in this study or the findings specified in this paper.

\section{Discussion}

Predisposing factors to vertebral osteomyelitis include diabetes mellitus, intravenous drug abuse, advancing age, malnutrition, an immunocompromised state, malignancy, and chronic steroid usage [2]. Unfortunately, there is often a delay in diagnosis of several weeks to months [3]. Patients may not present with fever or elevated WBC; however, elevated inflammatory parameters, such a high ESR and CRP, are much more consistent [3]. Conservative measures, including antibiotic therapy, bracing, and, in certain cases, bed rest, are sufficient for treatment in the majority of cases [3-4]. In one study of 360 patients who met inclusion criteria for VO, $86 \%$ were treated successfully with conservative measures [4]. Surgical treatment is required for those with neurologic deficit, spinal instability, vertebral body collapse, progressive spinal deformity, or infection unresponsive to antibiotic therapy and conservative measures [3-6]. Complication rates from those patients requiring surgical intervention may be as high as $60 \%$ with up to a $25 \%$ reoperation rate [4]. Long-term functional studies show that most surgical patients fail to reach normal SF-36 physical function scores and are often associated with an adverse outcome [6].

The most common causative pathogens for vertebral osteomyelitis include Staphylococcus aureus and Escherichia coli [1, 3]. Here, we present a case of vertebral osteomyelitis caused by Granulicatella adiacens. Only four previous cases of VO caused by G. adiacens have been reported in the literature [7-09]. In 2000, NVS were subtyped into four categories, including $G$. adiacens [10]. NVS are normal flora of the upper respiratory, urogenital, and gastrointestinal tracts of humans. NVS do not grow in common culture media, and the sensitivity of isolation by conventional methods is low. Thus, diagnosis of these infections is often difficult.

Furthermore, delayed treatment can lead to significant morbidity, as endocarditis is a common sequela [8].

Our patient presented with elevated WBC and ESR, but no fever. Given his recent history of trauma, his imaging was interpreted as possibly due to a mild compression fracture. His initial blood cultures were negative, and he was discharged without antibiotic therapy. The patient experienced significant progression of his VO in a four-week period, necessitating surgical intervention for stabilization.

\section{Conclusions}

Vertebral osteomyelitis is a common entity that is most commonly managed successfully with conservative measures, including antibiotics and immobilization with bracing. Delays in diagnosis may lead to progressive osseous destruction necessitating surgical intervention, which is associated with a reasonably high complication rate. Medical practitioners should maintain a heightened awareness for uncommon pathogens and presentations of VO to reduce 
such delays. Granulicatella adiacens is one such rare cause of VO that is often difficult to culture on traditional mediums and often requires PCR methodology for isolation. Early isolation and treatment of pathogens, such as G. adiacens, may decrease the need for surgical intervention.

\section{Additional Information \\ Disclosures}

Human subjects: Consent was obtained by all participants in this study. The authors report no conflict of interest concerning the materials or methods used in this study or the findings specified in this paper. Conflicts of interest: In compliance with the ICMJE uniform disclosure form, all authors declare the following: Payment/services info: All authors have declared that no financial support was received from any organization for the submitted work. Financial relationships: All authors have declared that they have no financial relationships at present or within the previous three years with any organizations that might have an interest in the submitted work. Other relationships: All authors have declared that there are no other relationships or activities that could appear to have influenced the submitted work.

\section{References}

1. Mylona E, Samarkos M, Kakalou E, Fanourgiakis P, Skoutelis A: Pyogenic vertebral osteomyelitis: a systematic review of clinical characteristics. Semin Arthritis Rheum. 2009, 39:10-17. 10.1016/j.semarthrit.2008.03.002

2. Gasbarrini AL, Bertoldi E, Mazzetti M, Fini L, Terzi S, Gonella F, Mirabile L, Barbanti Bròdano G, Furno A, Gasbarrini A, Boriani S: Clinical features, diagnostic and therapeutic approaches to haematogenous vertebral osteomyelitis. Eur Rev Med Pharmacol Sci. 2005, 9:53-66.

3. Butler JS, Shelly MJ, Timlin M, Powderly WG, O'Byrne JM: Nontuberculous pyogenic spinal infection in adults: a 12-year experience from a tertiary referral center. Spine (Phila Pa 1976). 2006, 31:2695-2700. 10.1097/01.brs.0000244662.78725.37

4. Miller JA, Achey RL, Derakhshan A, Lubelski D, Benzel EC, Mroz TE: Neurologic complications, reoperation, and clinical outcomes after surgery for vertebral osteomyelitis. Spine (Phila Pa 1976). 2016, 41:E197-204. 10.1097/BRS.0000000000001157

5. Chen WH, Jiang LS, Dai LY: Surgical treatment of pyogenic vertebral osteomyelitis with spinal instrumentation. Eur Spine J. 2007, 16:1307-16. 10.1007/s00586-006-0251-4

6. O'Daly BJ, Morris SF, O'Rourke SK: Long-term functional outcome in pyogenic spinal infection. Spine (Phila Pa 1976). 2008, 33:E246-53. 10.1097/BRS.0b013e31816b8872

7. Rosenthal O, Woywodt A, Kirschner P, Haller H: Vertebral osteomyelitis and endocarditis of a pacemaker lead due to Granulicatella (Abiotrophia) adiacens. Infection. 2002, 30:317-19. 10.1007/s15010-002-2104-3

8. Fukuda R, Oki M, Ueda A, Yanagi H, Komatsu M, Itoh M, Oka A, Nishina M, Ozawa H, Takagi A: Vertebral osteomyelitis associated with Granulicatella adiacens. Tokai J Exp Clin Med. 2010, 35:126-29.

9. Heath CH, Bowen SF, McCarthy JS, Dwyer B: Vertebral osteomyelitis and discitis associated with Abiotrophia adiacens (nutritionally variant streptococcus) infection. Aust N Z J Med. 1998, 28:663. 10.1111/j.1445-5994.1998.tb00667.x

10. Collins MD, Lawson PA: The genus Abiotrophia (Kawamura et al.) is not monophyletic: proposal of Granulicatella gen. nov., Granulicatella adiacens comb. nov., Granulicatella elegans comb. nov. and Granulicatella balaenopterae comb. nov. Int J Syst Evol Microbiol. 2000, 50:365-69. 10.1099/00207713-50-1-365 